\title{
El Papel de Variables Biopsicosociales en la Predicción del Factor Comportamental de la Insatisfacción Corporal. Un Modelo de Ecuaciones Estructurales
}

\author{
The Role of Biopsychosocial Variables in the Prediction of the Behavioral Factor of \\ Body Dissatisfaction. A Structural Equations Model
}

\author{
Jesús Enrique Peinado Pérez ${ }^{1}$, Humberto Blanco Vega², Martha Ornelas Contreras ${ }^{3}$, \\ Susana Ivonne Aguirre Vásquez ${ }^{4}$ y Natalia Solano-Pinto ${ }^{5}$
}

\begin{abstract}
Resumen
La insatisfacción corporal es un constructo multifactorial donde el componente comportamental se encuentra orientado a la modificación del cuerpo. Mediante modelos de ecuaciones estructurales se analizan los efectos directos e indirectos del género, los factores cognitivo-emocionales y perceptivo de la insatisfacción corporal, el porcentaje de grasa y el número de dietas sobre el factor comportamental de la insatisfacción corporal. Los participantes han sido 400 universitarios mexicanos 268 mujeres y 132 hombres, con una edad media de 20.55 años ( $\mathrm{DE}=1.83$ ). El modelo propuesto obtiene un ajuste satisfactorio. En particular, los factores malestar emocional y perceptivo son los más importantes en la explicación de la dimensión comportamental de la insatisfacción corporal. Además, dichos factores son mediadores entre el género, porcentaje de grasa y número de dietas. Se discuten las implicaciones de los hallazgos, tanto para la comprensión del constructo, como para la intervención y promoción de la salud.
\end{abstract}

Palabras clave: insatisfacción corporal, porcentaje de grasa, dietas, ecuaciones estructurales

\begin{abstract}
Body dissatisfaction is a multifactorial construct where the behavioral component is aimed at modifying the body. Through structural equation models, the direct and indirect effects of gender, cognitive-emotional and perceptual factors of body dissatisfaction, the percentage of body fat and numbers of diets on the behavioral factor of body dissatisfaction are analyzed. The sample comprised 400 Mexican college, 268 women and 132 men, with a mean age of 20.55 years-old $(\mathrm{SD}=1.83$ ). A satisfactory fit has been obtained. In particular, adding the direct and indirect effects (total effect), the emotional and perceptual factors are the most important in explaining the behavioral dimension of body dissatisfaction. In addition, these factors are mediators between gender, percentage of body fat and number of diets. The implications of the findings are discussed, both for the understanding of the construct, as well as for intervention and health promotion.
\end{abstract}

Keywords: body dissatisfaction, percentage of body fat, diets, structural equations

\footnotetext{
Financiación: Grupo de investigación de la Universidad de Castilla La Mancha, Salud, Educación y Sociedad (Mirada Crítica) con fondos aportados por el Plan Propio de Investigación de fecha 19 de febrero de 2020 (DOCM n 39 de 26/02/2020), cofinanciadas por el Fondo Europeo de Desarrollo Regional (FEDER), número 2020-GRIN- 29110.

${ }^{1}$ Profesor Investigador. Universidad Autónoma de Chihuahua. Facultad de Ciencias de la Cultura Física. DES Salud. Calle Escorza 900, CP 31000. Chihuahua, Chih. México. Tel.: 6144131507. Correo: jpeinado@uach.mx

${ }^{2}$ Profesor Investigador. Universidad Autónoma de Chihuahua. Facultad de Ciencias de la Cultura Física. DES Salud. Calle Escorza 900, CP 31000. Chihuahua, Chih. México. Tel.: 6144131507. Correo: hblanco@uach.mx

${ }^{3}$ Profesor Investigador. Facultad de Ciencias de la Cultura Física. Universidad Autónoma de Chihuahua. DES Salud. Calle Escorza 900, CP 31000. Chihuahua, Chih. México. Tel.: 6144131507. Correo: mornelas@uach.mx

${ }^{4}$ Profesor Investigador. Universidad Autónoma de Chihuahua. Facultad de Ciencias de la Cultura Física. DES Salud. Calle Escorza 900, CP 31000. Chihuahua, Chih. México. Tel.: 6144131507. Correo: siaguirre@uach.mx

${ }^{5}$ Doctora en Psicología. Contratada Doctora. Universidad Castilla La Mancha, España. Fábrica de Armas. Campus tecnológico. 45071, Toledo, España. Tel.: 925268800 ext: 5937. Correo: natalia.solano@uclm.es (Autora de Correspondencia)

Revista Iberoamericana de Diagnóstico y Evaluación - e Avaliação Psicológica. RIDEP · No59 · Vol.2 · 63-75 · 2021

ISSN: 1135-3848 print /2183-6051online
} 


\section{Introducción}

El desarrollo de la vivencia corporal constituye una experiencia subjetiva con un fuerte anclaje en los acontecimientos vividos en la infancia y en la adolescencia relacionándose con el desarrollo de la identidad y consolidación de la misma al final de la adolescencia y en la adultez emergente (Vartanian \& Hayward, 2020), siendo dinámica y cambiante en función de las experiencias y de los cambios corporales a lo largo de todo el ciclo vital (Rodríguez \& Alvis, 2015). De esta forma, el desarrollo de una imagen corporal positiva se relaciona con el desarrollo de la autoestima y calidad de vida (Nayir, et al., 2016) considerándose un factor protector y promotor de la salud. En la literatura la imagen corporal se considera un constructo multifactorial (Longo, 2016). Su desarrollo positivo se relaciona con experiencias donde se enfatiza la diversidad corporal otorgándole al cuerpo un valor funcional como vehículo que permite al ser humano vivir en plenitud (Dunaeva, Markey, \& Brochu, 2018).

Por el contrario, experiencias negativas relacionadas con el cuerpo, la presión sociocultural, un modelo estético irreal y asociado al éxito (Brown \& Tiggemann, 2016), contribuyen a la vivencia negativa de la imagen corporal, es decir, a sentir insatisfacción con el propio cuerpo. Tradicionalmente desde el modelo tripartito del desarrollo de la insatisfacción corporal, se contempla a los aspectos socioculturales, la influencia de la familia y de los iguales (Keery, Van den Berg, \& Thompson, 2004; Thompson, Heinberg, Altabe, \& Tantleff-Dunn,1999). La interiorización de un determinado cuerpo acompaña a los roles de género que es diferente para hombres y mujeres. Frágil, delgado para la mujer; musculado para el hombre (Karazsia, Murnen, \& Tylka, 2017). En ambos casos, un cuerpo irreal que promueve el consumo para poder alcanzarlo (Grogan, 2008), por ejemplo, vientre plano en el caso de las mujeres; cuerpo sin vello para los hombres. Aunque recientemente los hombres han sido incorporados al consumo y a la estética corporal, tradicionalmente la presión sociocultural hacia un cuerpo esbelto ha estado dirigida hacia la mujer, aspecto que se traduce en puntuaciones más elevadas en mujeres en comparación con los hombres (Latorre, López,
Izquierdo, \& García, 2018), llevando a algunos autores a eliminar de la evaluación o de la intervención a los jóvenes de sexo masculino (Castillo, Solano, \& Sepúlveda, 2018) considerando que la insatisfacción corporal es normativo entre las mujeres debido a la discrepancia permanente entre el cuerpo ideal y el cuerpo real (Carrard, Kruseman, \& MarquesVidal, 2018). Sin embargo, en los últimos años se encuentra una tendencia al aumento de insatisfacción entre los varones lo que está haciendo que se igualen las puntuaciones medias entre hombres y mujeres jóvenes (Holland \& Tiggemann, 2016).

Además de los aspectos comentados, existen otras variables que fomentan la insatisfacción corporal, en concreto, los jóvenes entre 18 y 24 años que intentan cambiar sus hábitos a través de una dieta no saludable desarrollan preocupaciones por su cuerpo y conductas propias de los trastornos alimentarios (Neumark-Sztainer, Wall, Larson, Eisenberg, \& Loth, 2011). Igualmente, las pérdidas de peso debido a conductas dietéticas no saludables conducen a internalizar obsesivamente el deseo de un cuerpo determinado contribuyendo a la aparición de conductas compulsivas tanto en hombres como en mujeres (Burnettea \& Mazzeo, 2020), siendo además un predictor de ganancia de peso a largo plazo (Lowe, Marti, Lesser, \& Stice, 2019).

Uno de los errores en la conceptualización de la insatisfacción corporal es reducir su definición al malestar con partes del cuerpo y/o con el cuerpo total (Thompson, 2004). Desde el modelo cognitivo-conductual, el malestar con la vivencia del propio cuerpo tiene manifestaciones multifactoriales, cognitivo, afectivo, perceptivo y comportamental que conforman el constructo de insatisfacción corporal (Alleva, Sheeran, Webb, Martijn, \& Miles, 2015). En la literatura se señala el componente cognitivo-emocional enmarcado en una dimensión actitudinal que englobaría cogniciones y emociones hacia el propio cuerpo con el planteamiento de modificar el cuerpo para perder peso, comparaciones con cuerpos esbeltos, focalización de la atención en zonas del cuerpo que más se aleja del ideal (caderas, tripa, brazos) y miedo a engordar, estando el plano afectivo relacionado con el malestar emocional como enfado, tristeza, ansiedad generado con la 
insatisfacción con el propio cuerpo (Thompson \& Schaefer, 2019).

El componente perceptivo de la insatisfacción corporal se refleja en la tendencia a la sobreestimación de la figura acompañada de cogniciones exageradas y negativas. En este sentido y desde el modelo cognitivo-conductual, se debe entender que este componente no se limita a la estimación de las dimensiones del cuerpo (Mölbert, et al., 2017) si no que existe una interacción entre las cogniciones, emociones y exageración acompañada de etiquetas negativas que conformarían el componente perceptivo (Pitron, Alsmith, \& deVignemont, 2018).

El componente comportamental se manifiesta a través de todas las conductas que la persona interpreta que puede cambiar su figura corporal, modificar su peso, disminuir el malestar o recuperar el control. Las interpretaciones subjetivas sobre qué es lo que puede modificar el peso hace que las conductas sean muy variadas, desde el uso compulsivo de la báscula o de la ropa con la percepción subjetiva de controlar el peso o la dimensión corporal o, todo lo contrario, la evitación del propio cuerpo (Bardone-Cone, Brownstone, Higgins, Fitzsimmons-Craft, \& Harney, 2013). En este sentido, algunos autores han mencionado las comprobaciones (body checking) que se realizan para verificar si las dimensiones corporales se han modificado (Trejger, Pereira, Brasiliano, Athanássios, \& Brunfentrinker, 2015). Las conductas desadaptativas para controlar el peso son consideradas por algunos autores como una alimentación emocional, conducta alimentaria dirigida a disminuir el malestar emocional ("emotional eating") lo que constituye una estrategia de afrontamiento inadecuada (Evers, Marijn Stok, \& de Ridder, 2010; Thayer, 2001).

Los componentes de la insatisfacción corporal, tal y como se han descrito, correlacionan y conforman el constructo de insatisfacción con la imagen corporal. Sin embargo, pueden mostrar cierta disincronía, es decir, una persona puede tener manifestaciones cognitivo- emocionales de insatisfacción corporal y no manifestar una tendencia a la sobreestimación, o sí, pero no realizar comportamientos para modificar o controlar el peso corporal (Thompson, 2004). Es como si fuera un gradiente $\mathrm{o}$ indicador de probabilidad de patología donde la dimensión que más poder predictivo tiene es el componente comportamental. En las manifestaciones de dicho componente aparecen conductas cercanas al comportamiento psicopatológico donde hay una conducta manipulativa con la finalidad de controlar el peso. Por dicha cercanía, es el componente de la insatisfacción corporal que más capacidad predictiva tiene en relación con los trastornos alimentarios. De esta forma, en otras investigaciones se obtuvo que aquellas jóvenes con una puntuación alta en el componente comportamental tenían 33.2 (odds ratio 33,2; IC $95 \%=15,1-68,3$ ) veces más probabilidad de tener un trastorno alimentario que aquellas jóvenes con puntuaciones bajas, además las pruebas del tamaño del efecto en el análisis de validez discriminante mostraba para el componente comportamental un valor de eta cuadrado de .47 , lo que indicaba su capacidad para clasificar correctamente la pertenencia a un grupo clínico y control (Solano-Pinto, CanoVindel, Blanco Vega, \& Fernández-Cézar, 2017). La diferenciación de los componentes de la imagen corporal, comprendiendo la interacción de los mismos, permitirá la identificación y evaluación de la insatisfacción corporal. De ahí, la recomendación de utilizar sistemas de evaluación específicos y de intervención para cada uno de sus componentes (Alleva, et al., 2015).

Asociado al miedo a engordar se encuentran las ideas, los debería, los propósitos que constituyen un planteamiento de cambio cognitivo y preparatorio en toda modificación de conducta. La relación entre el cuerpo esbelto y el éxito social y la discrepancia entre el ideal y el real no solo genera el deseo de un cuerpo delgado si no el rechazo a lo contrario, el cuerpo con sobrepeso y por tanto miedo a engordar. En este sentido, Wang et al. (2018) encuentran que la obsesión por adelgazar se relaciona con conductas para controlar el peso en jóvenes de sexo femenino, aunque no encontró esta relación en los jóvenes varones. Se supone que, a mayor peso, mayor insatisfacción corporal, sin embargo, algunos autores señalan que no necesariamente el peso y la obesidad se encuentra asociado a una imagen corporal negativa (Krch, 2004) pareciendo más importante la percepción del peso y la forma 
corporal que el índice de masa corporal real (Yan, Johnson, Harrell, Pulver, \& Zhang, 2015)

Además, el índice de masa corporal no es una medida que permita diferenciar porcentaje de grasa y de músculo. Debido a esta limitación, algunos autores recomiendan el uso del porcentaje de grasa como medida antropométrica (Davillas \& Benzeval, 2016). En este sentido, Ferrari et al. (2015) encuentra que el porcentaje de grasa es predictor de insatisfacción corporal debido al exceso peso en jóvenes y adolestentes chicos, con una odd ratio de 3.77. En la misma línea, Corseuil, Pelegrini, Bech y Petroski (2009) señalan que el nivel de adiposidad y estado nutricional se encuentra asociado a la imagen corporal en chicos y adolescentes. Por el contrario, algunos estudios han encontrado correlaciones positivas entre porcentaje de grasa e imagen corporal. En este sentido, los autores consideran que, aunque la personas tenga un elevado porcentaje de grasa puede sentir bienestar (Shuanglong, \& Guangye, 2018) y sentirse en forma, "fat but fit" (Duncan, 2010).

Las relaciones entre la realización de dietas no saludables y la insatisfacción corporal son complejas. Por una parte, la insatisfacción corporal puede llevar a la realización de dietas, pero también las conductas nutricionalmente inadecuadas ocasionan obsesividad y preocupación por el cuerpo (Craike et al., 2016). Por ello sería esperable que la dieta restrictiva acompañase a otros comportamientos propios del malestar con el propio cuerpo y, por tanto, predecirlas. En este sentido, los estudios indican un incremento en la realización de conductas inadecuadas para controlar el peso y la apariencia física en la sociedad occidental (Puhl et al., 2015). Quizás debido a que la internalización de un determinado peso y figura hace que la valoración de uno mismo se encuentre basada en el peso, así ambos aspectos, insatisfacción corporal y dicha internalización están asociados a la salud mental (O'Brien et al., 2016). Conviene tener en cuenta que la población universitaria es un grupo vulnerable al asumir por primera vez responsabilidades asociadas a los hábitos alimentarios, compra y preparación de alimentos (Téllez et al., 2015).

Aunque está descrita en la literatura revisada la importancia de la insatisfacción corporal, el poder predictivo del componente comportamental $\mathrm{y}$ el aumento de seguimiento de dietas, no se han encontrado modelos que establezca la relación de los distintos componentes. Por ello, los objetivos de este trabajo han sido construir un modelo predictivo sobre el componente comportamental de la insatisfacción corporal a través del número de dietas, porcentaje de grasa corporal y los diferentes componentes de la insatisfacción corporal.

\section{Método}

\section{Participantes}

Participaron en el estudio 400 jóvenes, 268 mujeres y 132 hombres estudiantes universitarios de México. Se trata de un estudio transversal, descriptivo y correlacional donde la muestra se obtuvo mediante un muestreo por conveniencia. Los participantes cursaban la Licenciatura en Psicología que se imparte en la Facultad de Psicología de la Universidad Autónoma de Yucatán. La edad de las participantes fluctuó entre los 17 y 27 años, una media de 20.55 y una desviación estándar de 1.83 años. Los criterios de inclusión fueron: ser estudiante de Psicología de la Universidad Autónoma de Yucatán y querer participar de forma voluntaria y anónima. Como criterios de exclusión se consideró: no ser estudiante de dicha universidad y no querer participar de forma voluntaria. Debido al procedimiento en la recogida de datos no hubo que desechar ningún cuestionario por lo que el tamaño muestral estuvo compuesto por los 400 estudiantes mencionados.

\section{Instrumento y variables}

Evaluación de la Insatisfacción con la Imagen Corporal (IMAGEN, Solano-Pinto \& Cano-Vindel, 2010), consta de 38 ítems que se responde con una escala de cinco puntos donde 0 (casi nunca o nunca) y 4 (casi siempre o siempre). La consistencia interna indicada por los autores es un alfa de Cronbach de .96 para el total de la prueba. Para este estudio se ha tenido en cuenta la versión abreviada (Solano-Pinto et al., 2017; Blanco, Solano-Pinto, Benavides, \& Ornelas 2017) donde se obtuvieron cinco factores: malestar emocional, miedo a engordar y planteamiento de cambio (como factores cognitivo emocional); factor 


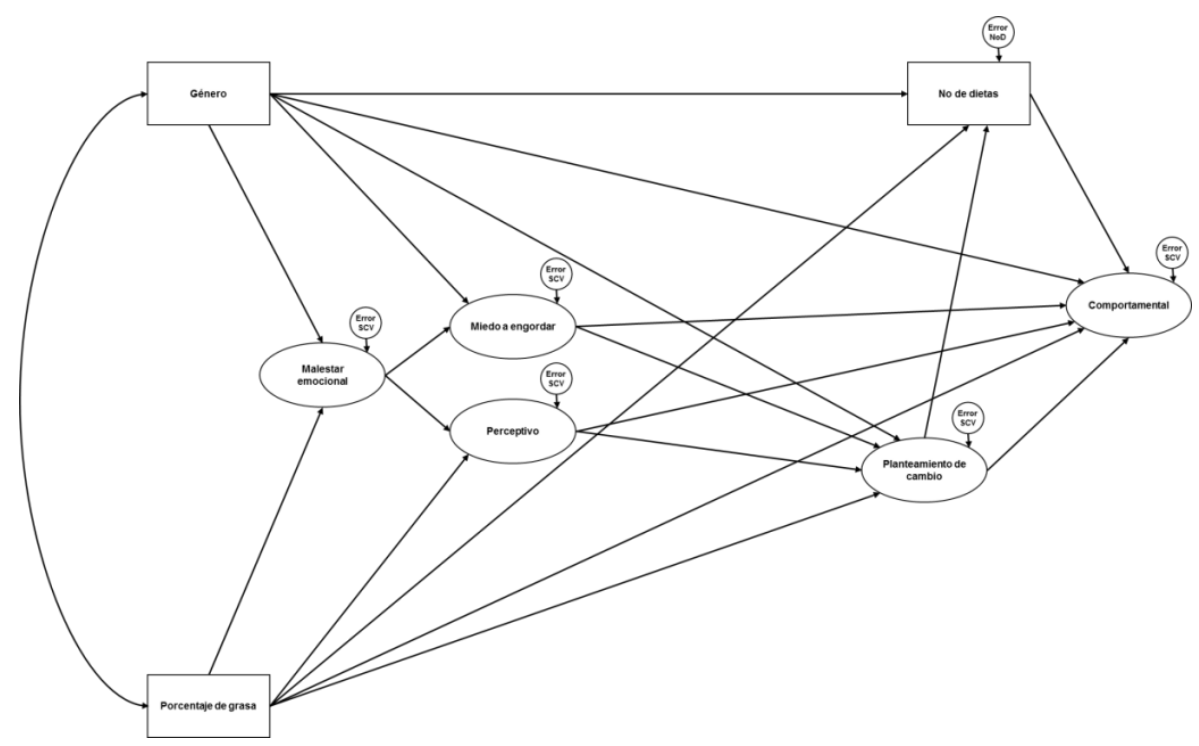

Figura 1. Modelo inicial propuesto para la dimensión comportamental de la insatisfacción corporal. Los indicadores de los factores no están representados por razones de simplicidad en la presentación

perceptivo y factor comportamental. Para esta investigación se realizaron los análisis estadísticos para comprobar las propiedades psicométricas del instrumento en la muestra evaluada, desechándose aquellos ítems que no presentaron cargas factoriales en su dimensión prevista.

Los resultados del análisis factorial confirmatorio para el modelo de 5 factores muestra un ajuste óptimo donde los cinco factores de este modelo explican en conjunto aproximadamente el $74 \%$ de la varianza. En concreto, para este estudio se han tenido en cuenta las siguientes escalas:

Componente cognitivo- emocional compuesto a su vez por las siguientes subescalas:

Planteamiento de cambio compuesto por los ítems 1,2 y 5 . Evalúa deseo de realizar dieta y actividad física con el objetivo de disminuir el peso. El coeficiente de alfa en la muestra del estudio es .843

Malestar emocional compuesto por los ítems $8,9,10,11$ y 19. La escala evalúa el grado de malestar emocional que ocasiona el cuerpo (enfado, sentimiento de inferioridad, tristeza), así como la comparación con otros cuerpos o los comentarios negativos. La consistencia interna en la muestra del estudio es de .901 .

Miedo a engordar, compuesta por los ítems 13,14 y 15 . Se valora el grado de miedo a perder el control sobre la figura y el peso, así como el miedo a engordar. El coeficiente Alfa de Cronbach es .900.
Componente perceptivo, con los ítems 26, 27, $28,29,30$ y 31 . La subescala evalúa la frecuencia de aparición de ideas y afirmaciones exageradas y peyorativas sobre la propia figura. El coeficiente Alfa de Cronbach es .884.

Componente comportamental, con los ítems 37 y 38. Evalúa la frecuencia con la que se manipula la comida implicando engaño y ocultación para disminuir la ingesta alimentaria con la finalidad de disminuir de peso. El coeficiente Alfa de Cronbach es .776.

Género donde el valor 0 representa a las mujeres y el 1 a los hombres.

Porcentaje de grasa. La medición se realizó a través de una báscula electrónica Tanita BC-418 Segmental Body Composition Analizer con 8 electrodos (4 para los pies y 4 para las manos) de BIA (Análisis de la Impedancia Bioeléctria) diseñada para personas entre 7 y 99 años, con una estatura entre $90 \mathrm{~cm}$ y $249 \mathrm{~cm}$, con capacidad de hasta 200 kilos y con incrementos de 100 gramos en peso y $1 \%$ de grasa, utilizando de 150 a 1200 ohmios. Tiene un rango de precisión de \pm 100 grs medidos a través de un método tetrapolar de bioimpedancia eléctrica con un espectro de medición de 50 khz.

Número de dietas: En los últimos dos años ¿Cuántas veces te has puesto a dieta para perder peso?

Como resultado de la revisión teórica se ha creado un modelo para predecir el componente comportamental de la insatisfacción corporal. Las 
variables contempladas en el modelo son los componentes cognitivo- emocionales y perceptivo, el género, el porcentaje de grasa corporal y el número de dietas (Figura 1). Del modelo se desprenden las hipótesis que a continuación se enuncian:

H1: Los factores emocionales (malestar emocional y el miedo a engordar) ejercen un efecto indirecto sobre la dimensión comportamental de la insatisfacción corporal a través de los factores cognitivo- emocionales y perceptivo de la insatisfacción corporal y el número de dietas.

$\mathrm{H} 2$ : La variable porcentaje de grasa ejerce un efecto indirecto sobre la dimensión comportamental de la insatisfacción corporal a través de los factores cognitivo- emocional y perceptivo y número de dietas.

H3: El género, el porcentaje de grasa y los factores cognitivo- emocionales y perceptivo y el número de dietas ejercen un efecto directo sobre la dimensión comportamental de la insatisfacción corporal.

H4: El factor perceptivo ejerce un efecto indirecto sobre la dimensión comportamental de la insatisfacción corporal a través de los factores cognitivo-emocional, y número de dietas

H5: El género ejerce un efecto indirecto en la dimensión comportamental a través de los componentes perceptivo y cognitivo- emocional de la insatisfacción corporal y del número de dietas.

\section{Procedimiento}

Se explicó la investigación a la Facultad de Psicología de la Universidad Autónoma de Yucatán (México). Posteriormente, se transmitieron los objetivos al alumnado y aquellos que decidieron voluntariamente participan firmaron el consentimiento informado garantizándose la confidencialidad de los datos. Completaron primero el Cuestionario IMAGEN y se tomaron las medidas de grasa corporal, se preguntó por el número de dietas, la edad y el sexo. La duración fue, aproximadamente, de 40 minutos. Al inicio de la sesión se hizo una pequeña introducción sobre la importancia de la investigación y se solicitó la máxima sinceridad. Al término de la sesión se les agradeció su participación. Además, los datos se recogieron de forma anonimizada, es decir, una vez el alumnado completaba los cuestionarios no se podía identificar ningún dato personal. De esta forma, se aseguraba el cumplimiento de la Declaración de Helsinki.

Posteriormente se procedió a recopilar los resultados por medio del módulo generador de resultados del editor de escalas versión 2.0 (31) (Blanco et al., 2013). Por último, los resultados obtenidos se analizaron mediante el paquete SPSS 18.0 y AMOS 21.0.

\section{Análisis de datos}

Con la finalidad de obtener los datos psicométricos respecto a la validez estructural se realizó un análisis confirmatorio mediante el programa AMOS 21 (Arbuckle, 2012). El método de estimación empleado fue el de Máxima Verosimilitud contrastando el modelo de tres y cinco factores del cuestionario IMAGEN, tal y como recomienda Thompson (2004). De esta forma, se compararon dos modelos de medida: el Modelo 1, modelo de tres factores acorde a la distribución original de los ítems dentro del cuestionario (Solano-Pinto \& Cano-Vindel, 2010) y el Modelo 2, modelo de cinco factores acorde a la composición factorial del cuestionario obtenida en otros estudios (Blanco et al., 2017; SolanoPinto et al., 2017). Posteriormente, con la finalidad de valorar la consistencia interna, se calculó la fiabilidad de cada uno de los factores del mejor de los modelos a través del Coeficiente Alfa de Cronbach (Elosua \& Zumbo, 2008; Nunnally \& Bernstein, 1995).

Los supuestos de normalidad y linealidad se comprobaron analizando los valores de asimetría y curtosis y los gráficos de dispersión matricial de las distintas variables. Después, se utilizó las ecuaciones estructurales para verificar el ajuste del modelo propuesto. A partir de la matriz de correlaciones se utilizó el método de estimación de máxima verosimilitud (ML). El ajuste de los modelos se comprobó a partir del Chi-cuadrado, el índice de bondad de ajuste (GFI), la raíz media cuadrática residual estandarizada (SRMR) y el error cuadrático medio de aproximación (RMSEA) como medidas absolutas de ajuste. El índice de bondad ajustado (AGFI), el Índice Tucker-Lewis (TLI) y el índice de bondad de ajuste comparativo (CFI) como medidas de ajuste 
incremental. La razón de Chi-cuadrado sobre los grados de libertad (CMIN/GL) y el Criterio de Información de Akaike (AIC) como medidas de ajuste de parsimonia (Byrne, 2010; Gelabert et al., 2011). Para el GFI, AGFI, TLI y CFI se estableció como umbral de aceptación valores superiores a .90 , e inferiores a .08 para el RMSEA y el SRMR (Byrne, 2010; Gelabert et al., 2011). Por último, se analizaron los efectos directos, indirectos y totales obtenidos entre las distintas variables contempladas en el modelo.

\section{Resultados}

En la Tabla 1 se resumen los valores de asimetría y curtosis de las distintas variables contempladas en el modelo inicial propuesto. La mayoría de las variables muestran valores de asimetría de \pm 2.00 y \pm 7.00 de curtosis, además, el índice multivariado de Mardia por encima del valor 70 indica alejamiento de la normalidad multivariada (Rodríguez \& Ruiz, 2008).

Tabla 1. Asimetría y curtosis para las variables observables del modelo inicial propuesto

\begin{tabular}{lcc}
\hline \multicolumn{1}{c}{ Variable } & AS & CU \\
\hline Género & .72 & -1.48 \\
Porcentaje de grasa & .01 & -.36 \\
Número de dietas & 3.99 & 21.72 \\
IMAGEN Ítem 1 & -.35 & -.20 \\
IMAGEN Ítem 2 & -.82 & .61 \\
IMAGEN Ítem 5 & -.71 & -.07 \\
IMAGEN Ítem 8 & .48 & -.17 \\
IMAGEN Ítem 9 & .60 & -.07 \\
IMAGEN Ítem 10 & .22 & -.93 \\
IMAGEN Ítem 11 & .50 & -.37 \\
IMAGEN Ítem 13 & .08 & -1.02 \\
IMAGEN Ítem 14 & .01 & -.86 \\
IMAGEN Ítem 15 & -.06 & -.91 \\
IMAGEN Ítem 19 & .26 & -.71 \\
IMAGEN Ítem 26 & 1.28 & .73 \\
IMAGEN Ítem 27 & 1.47 & 1.42 \\
IMAGEN Ítem 28 & 2.01 & 4.46 \\
IMAGEN Ítem 29 & 2.01 & 3.66 \\
IMAGEN Ítem 30 & 1.63 & 1.92 \\
IMAGEN Ítem 31 & 1.92 & 3.34 \\
IMAGEN Ítem 37 & 3.96 & 16.28 \\
IMAGEN Ítem 38 & 3.59 & 13.74 \\
\multicolumn{1}{c}{ Índice multivariado de Mardia } & & 166.73 \\
\hline Nota AS=asimetría: CU=curtosis & &
\end{tabular}

Los resultados globales del análisis factorial confirmatorio (GFI .925; RMSEA .049; CFI .965) para el modelo inicial propuesto indican que su ajuste es aceptable (Tabla 2). Sin embargo, dado que tres de las relaciones esperadas no resultaron significativas (Figura 2) se realizó una reespecificación del modelo inicial eliminando las relaciones no significativas: género > dimensión comportamental de la insatisfacción corporal, miedo a engordar > dimensión comportamental de la insatisfacción corporal y planteamiento de cambio > dimensión comportamental de la insatisfacción corporal (Figura 3).

Tras la eliminación de las vías (paths) no significativas, el modelo final GFI .924; RMSEA .049; CFI .965) sigue presentando un ajuste aceptable (Tabla 2).

Finalmente, se observa que ambos modelos explican un porcentaje de varianza similar en la variable criterio. Tanto el modelo inicial propuesto como el modelo final explican cerca del $40 \%$ de la varianza en la dimensión comportamental de la insatisfacción corporal (Figura 2 y 3 ).

Analizando individualmente los coeficientes de regresión para cada una de las vías propuestas en el modelo final (Figura 3), se observó que la totalidad de las relaciones propuestas obtienen significación cuando menos a un nivel $p<.05$.

Los efectos directo más elevados fueron los que produce el factor malestar emocional en el factor miedo a engordar $(\beta=.66, p<.001)$ y en el factor perceptivo $(\beta=.65, p<.001)$. De manera similar destaca el efecto del factor miedo a engordar sobre el factor planteamiento de cambio. Finalmente, en la Figura 3 se puede observar que, salvo tres relacionados con la variable género y dos de la variable porcentaje de grasa, la mayoría de los efectos directos son positivos.

Por otro lado, los resultados (Tabla 3) muestran que el género y el porcentaje de grasa influyen indirectamente de forma significativa en la variable número de dietas y los factores miedo a engordar, perceptivo, planteamiento de cambio y la dimensión comportamental de la insatisfacción corporal. Por su parte los factores miedo a engordar y perceptivo ejercen un efecto indirecto sobre la dimensión comportamental de la insatisfacción corporal y el número de dietas, mientras que el factor malestar emocional lo tiene hacia la variable número de dietas y los factores comportamental (dimensión comportamental de la insatisfacción corporal) y planteamiento de cambio; 
Tabla 2. Índices absolutos, incrementales y de parsimonia de los modelos inicial y final

\begin{tabular}{|c|c|c|c|c|c|c|c|c|c|}
\hline \multirow[b]{2}{*}{ Modelo } & \multicolumn{4}{|c|}{ Índices absolutos } & \multicolumn{3}{|c|}{ Índices incrementales } & \multicolumn{2}{|c|}{ Índices de parsimonia } \\
\hline & $\chi^{2}$ & GFI & RMSEA & SRMR & AGFI & TLI & CFI & CMIN/DF & AIC \\
\hline Inicial & $366.362 *$ & .925 & .049 & .044 & .898 & .957 & .965 & 1.970 & 500.362 \\
\hline Final & $369.449 *$ & .924 & .049 & .044 & .898 & .957 & .965 & 1.95 & 497.449 \\
\hline
\end{tabular}

divided by degrees of freedom; AIC=Akaike information criterion

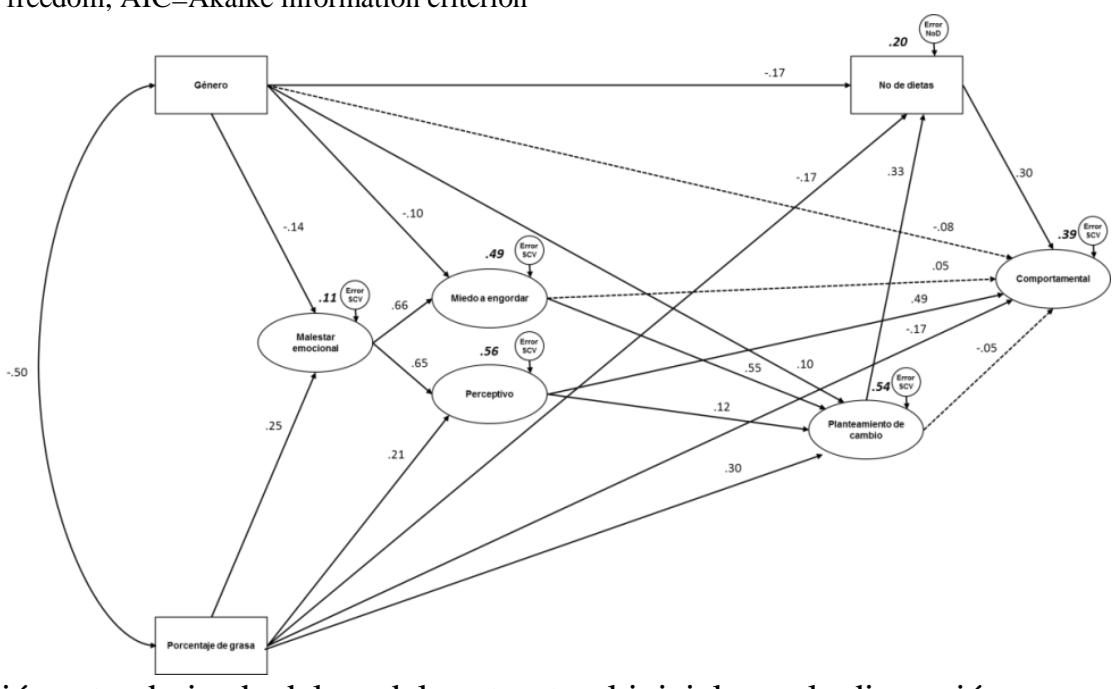

Figura 2. Solución estandarizada del modelo estructural inicial para la dimensión comportamental de la insatisfacción corporal. Todos los parámetros están estandarizados. Las líneas discontinuas representan paths no significativos

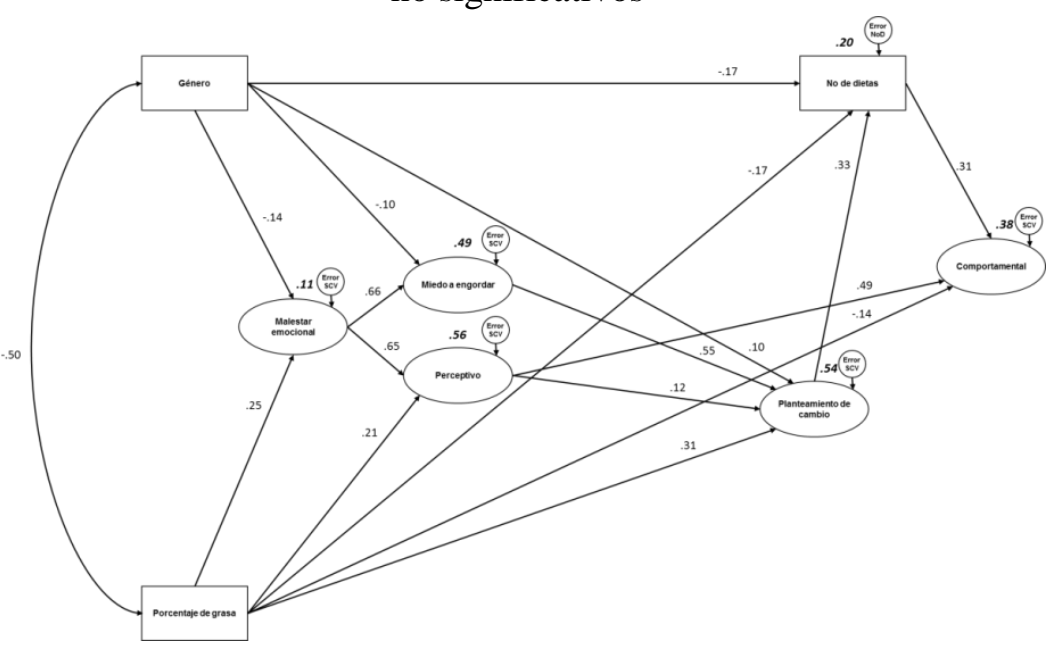

Figura 3. Solución estandarizada del modelo estructural final para la dimensión comportamental de la insatisfacción corporal. Todos los parámetros están estandarizados

y este último un efecto indirecto también sobre el factor comportamental.

\section{Discusión}

Una cuestión poco estudiada en la literatura revisada es la relación entre los componentes de la insatisfacción corporal en la explicación del componente comportamental, aspecto que se considera importante por su capacidad predictiva en las alteraciones alimentarias (Solano-Pinto et al., 2017). El modelo obtenido en esta investigación es novedoso ya que no se han encontrado estudios similares en muestras sin patología clínica con los que poder contrastar los resultados. En particular, sumando los efectos directos e indirectos (efecto total), los factores malestar emocional y perceptivo son los más importantes en la explicación de las diferencias o varianza de la dimensión comportamental de la insatisfacción corporal. Así, el malestar emocional, la tristeza, el enfado, el sentimiento de inferioridad, puede predecir el componente comportamental a través de variables mediadoras, bifurcándose en dos vías fundamentales, una 
Tabla 3. Efectos directos e indirectos estandarizados entre las variables consideradas en el modelo estructural final

\begin{tabular}{|c|c|c|c|c|c|c|c|c|}
\hline & & $\begin{array}{c}\text { Porcentaje de } \\
\text { grasa }\end{array}$ & Género & $\begin{array}{c}\text { Malestar } \\
\text { emocional }\end{array}$ & $\begin{array}{l}\text { Miedo a } \\
\text { engordar }\end{array}$ & Perceptivo & $\begin{array}{c}\text { Planteamiento } \\
\text { de cambio }\end{array}$ & $\begin{array}{c}\text { Número de } \\
\text { dietas }\end{array}$ \\
\hline $\begin{array}{l}\text { Malestar } \\
\text { emocional }\end{array}$ & $\begin{array}{l}\text { Directo } \\
\text { Indirecto }\end{array}$ & .25 & -.14 & & & & & \\
\hline Miedo a & Directo & & -.10 & .66 & & & & \\
\hline engordar & Indirecto & .16 & -.09 & & & & & \\
\hline Perceptivo & $\begin{array}{l}\text { Directo } \\
\text { Indirecto }\end{array}$ & $\begin{array}{l}.21 \\
.16\end{array}$ & -.09 & .65 & & & & \\
\hline $\begin{array}{l}\text { Planteamiento } \\
\text { de cambio }\end{array}$ & $\begin{array}{l}\text { Directo } \\
\text { Indirecto }\end{array}$ & $\begin{array}{l}.31 \\
.13\end{array}$ & $\begin{array}{l}.10 \\
-.12\end{array}$ & .44 & .55 & .12 & & \\
\hline $\begin{array}{l}\text { Número de } \\
\text { dietas }\end{array}$ & $\begin{array}{l}\text { Directo } \\
\text { Indirecto }\end{array}$ & $\begin{array}{r}-.17 \\
.22\end{array}$ & $\begin{array}{l}-.17 \\
-.02\end{array}$ & .27 & .18 & $\begin{array}{l}.19 \\
.04\end{array}$ & .33 & \\
\hline $\begin{array}{l}\text { Dimensión } \\
\text { comportamental }\end{array}$ & $\begin{array}{l}\text { Directo } \\
\text { Indirecto }\end{array}$ & $\begin{array}{l}-.14 \\
.20\end{array}$ & -.10 & .40 & .06 & $\begin{array}{l}.49 \\
.07\end{array}$ & .10 & .31 \\
\hline
\end{tabular}

donde la mediadora es el componente perceptivo, y otra, donde las mediadoras son el miedo a engordar y el planteamiento de cambio. Este modelo podría implicar un gradiente de insatisfacción que ayuda a entender la relación entre los componentes, aspecto fundamental (Thompson, 2004) para profundizar en la evaluación y en una intervención específica en cada uno de dichos componentes, tal y como recomiendan Alleva et al. (2015).

Otras variables que se han utilizado en la predicción del componente comportamental de la insatisfacción corporal han sido el porcentaje de grasa, el número de dietas y el género. Según indica la literatura revisada el porcentaje de grasa es una de las formas más idónea para valorar la obesidad (Davillas \& Benzeval, 2016). Los resultados son contradictorios en la relación entre grasa corporal e insatisfacción corporal ya que, aunque tradicionalmente se ha asociado la obesidad a la insatisfacción (Corseuil et al., 2009; Ferrari et al., 2015), algunas investigaciones muestran que con obesidad una persona puede tener una imagen corporal positiva, sentirse en forma y tener bienestar (Duncan, 2010; Shuanglong \& Guangye, 2018). Quizás este enfoque explicaría la relación negativa obtenida en este estudio entre porcentaje de grasa y componente comportamental que implicaría que a mayor porcentaje de grasa, menor tendencia a manipular de manera engañosa la comida y menor probabilidad a realizar dietas con la finalidad de perder peso, bien porque la persona no lo estima necesario, siguiendo la concepción de Duncan (fat but fit) o porque no perciben la necesidad de modificar su obesidad, aspecto que no se puede concretar con los resultados obtenidos en esta investigación. En cualquier caso, los hallazgos se muestran acorde con las afirmaciones de distintos investigadores donde se recalca la mayor importancia de sentir malestar con el cuerpo con cierta independencia al peso real (Krch, 2004; Yan et al., 2015).

Siguiendo la misma línea, se destaca a las variables mediadoras en las relaciones indirectas y positivas que se establecen entre el porcentaje de grasa y el factor comportamental. Estos mediadores pueden tener varias vías, tal y como se muestran en los resultados, donde se resalta el papel del malestar emocional ocasionado por el cuerpo y de la percepción exagerada del mismo. Las conductas inadecuadas podrían tener la finalidad de disminuir el malestar emocional que ocasiona la vivencia negativa del cuerpo (Trejger et al., 2015), conductas que pueden conformar lo que diferentes autores denominan "emotional eating" o alimentación emocional (Evers et al., 2010; Thayer, 2001). En este estudio, se puede concluir que el porcentaje de grasa hace más probable la aparición de comportamientos no saludables propios del componente comportamental de la imagen corporal a través del número de dietas y planteamiento de cambio constituyendo, el número de dietas, una variable mediadora. Estos resultados se encuentran en la misma línea que aquellas investigaciones que han encontrado que los jóvenes que cambian sus hábitos dietéticos por motivos estéticos desarrollan más preocupaciones por el cuerpo y conductas no saludables (Burnettea \& Mazzeo, 2020; Neumark-Sztainer et al., 2011), por lo que la realización de dietas no saludables se convierte en una variable en la que hay que intervenir entre otros, para evitar la ganancia de peso a largo plazo 
(Lowe et al., 2019). En la literatura, dichas conductas aparecen asociadas a los estereotipos negativos relacionados con la obesidad que fomentan la interiorización de un determinado cuerpo (O’Brien, et al., 2016) y que podría explicar el incremento en la prevalencia de conductas inadecuadas para controlar el peso y la figura (Puhl et al., 2015).

¿Qué papel juega el género en las interacciones comentadas? Según el modelo propuesto no tiene una relación directa con el factor comportamental quizás debido al aumento de insatisfacción corporal entre los hombres (Holland \& Tiggemann, 2016). Sin embargo, se establecen relaciones negativas entre porcentaje de grasa y género y entre género y dietas, aspecto que significa que las mujeres tienen más grasa corporal y realizan un mayor número de dietas que los hombres, resultado coincidente con otros estudios (Carrard et al., 2018; Latorre et al., 2018). Aunque no se establecen relaciones directas con el componente comportamental, sí aparecen asociaciones indirectas siendo los mediadores los aspectos emocionales y perceptivos de la imagen corporal, es decir, las relaciones comentadas entre porcentaje de grasa y componente comportamental se cumple con mayor probabilidad en jóvenes de sexo femenino lo que puede responder a la mayor presión sociocultural dirigida a las mujeres. Esta cuestión es importante porque no es cuestión de normalizar la insatisfacción corporal o la realización de dietas en las mujeres, si no de enfatizar la necesidad de transmitir otros ideales de belleza basadas en la funcionalidad y diversidad del cuerpo, tal y como destacan distintos estudios (Dunaeva et al., 2018), sin olvidar en la evaluación y acciones preventivas, al sexo masculino (Castillo et al., 2018).

Por último, a la luz de los resultados obtenidos se pueden extraer las siguientes conclusiones:

En primer lugar, el conjunto de variables explica en buena medida el factor comportamental de la insatisfacción corporal de los jóvenes universitarios. Así, las variables contempladas en el modelo alcanzan a explicar cerca del $40 \%$ de la varianza total del factor comportamental de la insatisfacción corporal, lo que parece revelar la importancia del conjunto de variables en la predicción y explicación de esta dimensión de la insatisfacción corporal en los universitarios.

Estos hallazgos tienen un carácter novedoso ya que en la revisión realizada no se han encontrado modelos explicativos entre los diferentes componentes de la insatisfacción corporal en la explicación del componente comportamental. Los resultados de esta investigación muestran cómo el número de dietas y el porcentaje de grasa son predictoras cuando los factores emocionales y perceptivos de la insatisfacción corporal actúan como mediadoras. Estos hallazgos podrían tener implicaciones en los programas para la promoción de la salud entre jóvenes. En este sentido, se podría fomentar la realización de dieta, entendida como alimentación equilibrada, dirigida al cuidado de la salud y la funcionalidad del cuerpo, eliminando el objetivo de modificar el peso y/o la forma corporal. De esta manera, se estaría disminuyendo el malestar emocional, a la vez que, la realización de actividad física y alimentación equilibrada tendrían una finalidad orientada al cuidado del cuerpo con independencia de la grasa corporal. Dichos aspectos estarían afianzando una imagen corporal positiva entre los jóvenes de ambos sexos.

En esta investigación existen varias limitaciones que conviene tener en cuenta con el fin de ser cauteloso en la generalización de los resultados. Se destacan las características muestrales, las franjas etarias y la falta de control de variables extrañas. Como líneas de actuación futura, será conveniente seleccionar la muestra de manera aleatoria entre diferentes ámbitos, así como el control de distintas variables que pudieran ser predictoras del componente comportamental de la insatisfacción corporal. Además, se podría utilizar un diseño longitudinal que permitiera conocer cómo evoluciona la relación entre las variables a lo largo de la adolescencia y adultez emergente. Igualmente, sería de interés realizar propuestas para incluir programas en el ámbito universitario, grupo considerado vulnerable (Téllez et al., 2015), que faciliten el desarrollo de la imagen corporal positiva. 


\section{Referencias}

Alleva, J. M., Sheeran, P., Webb, T. L., Martijn, C., \& Miles, E. A. (2015). Meta-analytic review of stand-alone interventions to improve body image. PLoS One, 10(9), e0139177.

doi: 10.1371/journal.pone.0139177.

Arbuckle, J. R. (2012). AMOS users guide version 21.0. Chicago, IL: Marketing Department, SPSS Incorporated.

Blanco, H., Ornelas, M., Tristán, J. L., Cocca, A., Mayorga-Vega, D., López-Walle, J., \& Viciana, J. (2013). Editor for creating and applying computerise surveys. Procedia Social and Behavioral Sciences, 106, 935940. doi:10.1016/j.sbspro.2013.12.105

Blanco, J. R., Solano-Pinto, N., Benavides, E. V., \& Ornelas, M. (2017). Composición e invarianza factorial del cuestionario IMAGEN en adolescentes mexicanos y españoles. Cuadernos de Psicología del Deporte, 17, 35-44.

Brown, Z., \& Tiggemann, M. (2016). Attractive celebrity and peer images on Instagram: Effect on women's mood and body image. Body Image, 19, 37-43. doi:10.1016/j.bodyim.2016.08.007.

Burnettea, C. B., \& Mazzeo, S. E. (2020). Examining the contribution of weight-bias internalization to the associations between weight suppression and disordered eating in undergraduates. Eating Behaviors, 37, 101392. doi:10.1016/j.eatbeh.2020.101392

Byrne, B. M. (2010). Structural equation modeling with AMOS: Basic concepts, applications and programming. New York, NY: Routledge.

Carrard, I, Kruseman, M., \& Marques-Vidal, P. (2018). Desire to lose weight, dietary intake and psychological correlates among middleaged and older women. The CoLaus study. Preventive Medicine, 113, 41-50. doi:10.1016/j.ypmed.2018.05.011.

Castillo, I., Solano, S., \& Sepúlveda, A. R. (2018). Validación mexicana de la Escala de Actitudes Masculinas sobre el Cuerpo en estudiantes universitarios varones. Revista Iberoamericana de Diagnóstico y Evaluación
- e Avaliação Psicológica, 46(1), 51-65. doi:10.21865/RIDEP46.1.04.

Craike, M. Young, J.A., Symons, C. M., Pain, M. D. Harvey, J. T., Eime, R. M., \& Payne, W.R. (2016). Trends in body image of adolescent females in metropolitan and nonmetropolitan regions: A longitudinal study. BMC Public Health, 16, 1143. doi:10.1186/s12889-016-3815-1.

Corseuil, M. W., Pelegrini, A., Beck, C., \& Petroski, E. L. (2009). Prevalência de insatisfação com a imagem corporal e sua associação com a inadequação nutricional em adolescentes. Revista de Educação Física, 20, 25-31.

Davillas, A., \& Benzeval, M. (2016). Alternative measures to BMI: Exploring income-related inequalities in adiposity in Great Britain. Social Science \& Medicine, 166, 223-232. doi:10.1016/j.socscimed.2016.08.032

Dunaev, J., Markey, Ch. H., \& Brochu, P. M. (2018). An attitude of gratitude: The effects of body-focused gratitude on weight bias internalization and body image. Body Image, 25, 9-14. doi:10.1016/j.bodyim.2018.01.006.

Duncan, G. E. (2010). The "fit but fat" concept revisited: Population based estimates using NHANES. International Journal of Behavioral Nutrition and Physical Activity, 7, 47. doi:10.1186/1479-5868-7-47.

Elosua, P., \& Zumbo, B.D. (2008). Coeficientes de fiabilidad para escalas de respuesta categórica ordenadas. Psicothema, 20, 896901.

Evers, C., Marijn Stok, F., \& de Ridder, D. T. D. (2010). Feeding your feelings: Emotion regulation strategies and emotional eating. Personality and Social Psychology Bulletin, 36, 792-804. doi:10.1177/0146167210371383.

Ferrari, E. P., Minatto, G., Berria, J., dos Silva, S. F., Fidelix, Y. L., Ribeiro, R. R., Santos, K. D., \& Petroski, K. L. (2015). Body image dissatisfaction and anthropometric indicators in male children and adolescents. European Journal of Clinical Nutrition, 69, 1140-1144 doi:10.1038/ejen.2014.252

Gelabert, E., García-Esteve, L., Martín-Santos, R., Gutiérrez, F., Torres, A., \& Subirà, S. (2011). Psychometric properties of the Spanish version of the Frost Multidimensional 
Perfectionism Scale in women. Psicothema, 23(1), 133-139.

Grogan, S. (2008). Body image: Understanding body dissatisfaction in men, women and children. New York: Routledge.

Holland, G., \& Tiggemann, M. (2016). A systematic review of the impact of the use of social networking sites on body image and disordered eating outcomes. Body Image, 17, 100-110. doi:10.1016/j.bodyim.2016.02.008.

Karazsia, B. T., Murnen, S. K., \& Tylka, T. L. (2017). Is body dissatisfaction changing across time? A cross-temporal meta-analysis. Psychological Bulletin, 143, 293-320. doi:10.1037/bul0000081

Keery, H., Van den Berg, P., \& Thompson, J. K. (2004). An evaluation of the tripartite influence model of body dissatisfaction and eating disturbance in adolescent girls. Body Image, 1, 237-251.

Krch FD. (2004). Eating disorders - risk factors. Psychiatrische Praxis, 5(1),14-6.

Latorre, P. A., López, R., Izquierdo, T., \& García, F. (2018). La satisfacción corporal en adultos españoles, Influencia del sexo, edad y estado ponderal. Revista Iberoamericana de Diagnóstico y Evaluación - e Avaliação Psicológica, 47(2), 83-94. doi:10.21865/RIDEP47.2.06

Longo, M. R. (2016). Types of body representation. In J. Coello \& M.J. Fischer (eds), Foundations of embodied cognition: Perceptual and emotional embodiment (pp117-134). London: Routledge.

Lowe, M. R., Marti, C. N., Lesser, E. L., \& Stice, E. (2019). Weight suppression uniquely predicts body fat gain in first-year female college students. Eating Behaviors, 32, 60-64. doi:10.1016/J.EATBEH.2018.11.005.

Nayir, T., Uskun, E., Yürekli, M. V., Devran, H., Çelik, A., \& Okyay, R. A. (2016). Does body image affect quality of life?: A population based study. PLoS One, 11, e0163290. doi:10.1371/journal.pone.0163290.

Neumark-Sztainer, D., Wall, M., Larson, N. I., Eisenberg, M. E., \& Loth, K. (2011). Dieting and disordered eating behaviors from adolescence to young adulthood: Findings from a 10-year longitudinal study. Journal of the American Dietetic Association, 111(7), 1004-1011. doi:10.1016/j.jada.2011.04.012.

Nunnally, J.C., \& Bernstein, I.H. (1995). Teoría Psicométrica. México: McGraw-Hill.

Mölbert, S. C., Klein, L., Thaler, A., Mohler, B. J., Brozzo, C., \& Martus, P., et al. (2017). Depictive and metric body size estimation in anorexia nervosa and bulimia nervosa: A systematic review and meta-analysis. Clinical Psychology Review, 57, 21-31. doi:10.1016/j.cpr.2017.08.005.

O’Brien, K. S., Latner, J. D., Puhl, R. M., Vartanian, L. R., Giles, C., \& Griva, K., et al. (2016). The relationship between weight stigma and eating behavior is explained by weight bias internalization and psychological distress. Appetite, 102, 70-76. doi:10.1016/j.appet.2016.02.032

Pitron, V., Alsmith, A., \& deVignemont, F. (2018). How do the body schema and the body image interact. Consciousness and Cognition, 65, 352-358.

doi:10.1016/j.concog. 2018.08.007.

Puhl, R. M., Latner, J. D., O’Brien, K., Luedicke, J., Danielsdottir, S., \& Forhan, M. (2015). A multinational examination of weight bias: Predictors of anti-fat attitudes across four countries. International Journal of Obesity, 39, 1166-1173. doi:10.1038/ijo.2015.32.

Rodríguez, D. F., \& Alvis, K. M. (2015). Overview of the body image and its implications in sport. Revista de la Facultad de Medicina, 63, 279-287. doi:10.15446/revfacmed.v63n2.49387.

Rodríguez, M. N., \& Ruiz, M. A. (2008). Atenuación de la asimetría y de la curtosis de las puntuaciones observadas mediante transformaciones de variables: Incidencia sobre la estructura factorial. Psicológica, 29, 205-227.

O’Brien, K. S., Latner, J. D., Puhl, R. M., Vartanian, L. R., Giles, C., Griva, K., \& Carter, A. (2016). The relationship between weight stigma and eating behavior is explained by weight bias internalization and psychological distress. Appetite, 102, 70-76. doi:10.1016/j.appet.2016.02.032.

Solano-Pinto, N., \& Cano-Vindel, A. (2010). IMAGEN Evaluación de la Insatisfacción con la Imagen Corporal. Madrid: TEA Ediciones. 
Solano-Pinto, N., Cano-Vindel, A., Blanco Vega, H., \& Fernández-Cézar, R. (2017). Datos psicométricos de la versión abreviada del cuestionario IMAGEN; evaluación de la insatisfacción corporal. Nutrición Hospitalaria, 34, 952-960. doi:10.20960/nh.695.

Shuanglong, L., \& Guangye, H. (2018). Laugh and grow fat: Happiness affects body mass index among urban Chinese adults. Social Science \& Medicine, 208, 55-63. doi:10.1016/j.socscimed.2018.05.008.

Téllez, E., Castillo, N., García, S., Yagüe, I., Requena, M., Olmedilla Y. L., Arnoriaga, M., \& Andía, V. M. (2015). Satisfacción con la propia imagen corporal en una población de estudiantes universitarios de la Comunidad de Madrid. Nutrición Hospitalaria, 31(3), 14231426. doi:10.3305/nh.2015.31.3.8349

Thayer, R. E. (2001). Calm energy: How people regulate mood with food and exercise. New York, NY: Oxford University Press.

Thompson, B. (2004). Exploratory and Confirmatory Factor Analysis. Understanding concepts and applications. Washington, D C: American Psychological Association.

Thompson, J.K. (2004). The (mis) measurement of body image: ten strategies to improve assessment for applied and research purposes. Body Image, 1, 7-14. doi:10.1016/S1740-1445(03)00004-4.

Thompson, J. K., Heinberg, L. J., Altabe, M., \& Tantleff-Dunn, S. (1999). Exacting beauty: Theory, assessment, and treatment of body image disturbance. Washington, DC: American Psychological Association.

Thompson, J. K., \& Schaefer, L. M. (2019). Thomas F. Cash: A multidimensional innovator in the measurement of body image; Some lessons learned and some lessons for the future of the field. Body Image, 31,198203. doi:10.1016/j.bodyim.2019.08.006.

Trejger, A., Pereira, L., Brasiliano, S., Athanássios, T., \& Brunfentrinker, P. (2015). Body checking and eating cognitions in Brazilian outpatients with eating disorders and non psychiatric controls. Eating Behavior, 19, 184-187. doi:10.1016/j.eatbeh.2015.09.009

Vartanian, L. R., \& Hayward, L. E. (2020). Dimensions of internalization relevant to the identity disruption model of body dissatisfaction. Body Image, 32, 1-4. doi:10.1016/j.bodyim.2019.10.008.

Wang, K., Liang, R., Ma, Z. L., Chen, J., Cheung, E. F. C., Roalf, D. R., Gur, R. C., \& Chan, R. C. K. (2018). Body image attitude among Chinese college students. PsyCh Journal, 7, 31-40. doi:10.1002/pchj.200.

Yan, F., Johnson, A. M., Harrell, A., Pulver, A., \& Zhang, J. (2015). Perception or reality of body weight: Which matters the most to adolescents' emotional wellbeing? Current Psychiatry Reviews, 11(2),130-46. 